\title{
Tools for Automatic Wound Healing Evaluation
}

\author{
by A. Nowakowski*, M. Kaczmarek*, M. Moderhak*, P. Siondalski**, Ł. Woś ${ }^{* *}$ \\ * Gdansk University of Technology, WETI, Department of Biomedical Engineering, Narutowicza 11/12, \\ 80-233 Gdansk, Poland, antowak@biomed.eti.pg.gda.pl \\ ** Medical University of Gdansk, Clinic of Cardiac \& Vascular Surgery, Debinki 7, 80-211 Gdansk, Poland
}

\begin{abstract}
Final results of the research project devoted to development of a new objective and quantitative method of post cardiosurgery wound healing diagnostics, already preliminary discussed at the QIRT 2014 Conference [1], is presented. We assumed that thermal processes should be effective in subtle description of temporal changes of tissue thermal properties after surgical interventions. To prove this assumption more than 400 patients after cardiosurgery intervention on the open heart participated in clinical experiments using IR thermal cameras as diagnostic instrumentation. We applied both - classical thermal image capture as well as active dynamic thermography method ADT - for analysis of thermal processes existed in the vicinity of the postoperative wound. Here we concentrate on description of technical issues of the developed instrumentation and procedures applied in the research. Discussion is devoted to the problem of standardization of measurement conditions, possible hardware solutions to be applied in clinics and necessary procedures of data acquisition and processing.
\end{abstract}

\section{Introduction}

This is the summary of the research project financed by the Polish National Science Centre devoted to evaluation of cardio-surgery wound healing based on analysis of thermal tissue properties using QIRT approach. We already presented preliminary results of this project at the QIRT Conference in Bordeaux in 2014 [1]. To conclude our experience here we discuss all technical and practical issues necessary to make experimental part of the project operational in clinical environment. We mention some medical requirements, too, as medical and legal problems may be not less important than technology in terms of successful implementation of new diagnostic procedures.

The presentation is structured into parts devoted to:

a/ basics of the method;

b/ hardware requirements with special notice on Active Dynamic Thermography based on cryotherapy unit for cooling; also on matching IR and RGB camera images;

c/ software tools for data capture, here most important are driving signals for cameras and cooling devices and registration condition data;

d/ software tools for data analysis as: a proper database; algorithms of image corrections and determination of proper ROI; generation of parametric images; extraction of diagnostic information from multimodality data etc.

In conclusion we declare a successful implementation of the method in clinical environment. This is the first quantitative approach allowing clear documentation of post cardiosurgery wound healing progress.

\section{The aim of the research}

Basic progress in medicine nowadays is mainly due to implementation of new medical technology in clinical practice. New imaging technologies open possibility of objective evaluation and diagnostics of processes existing inside the human body. Better understanding of physiology allows for positive feedback leading to matured treatment procedures and constant improvement of medical services. It is well known, that any malfunction in physiology is directly evidenced by changes of temperature, formation of cold or hot spots at the surface of skin, depending if it is due to decreased or increased metabolism and blood flows.

One of important but still not fully accepted in medical practice modalities is IR-thermal imaging. Our long lasting experience in development of this modality convinced us and cooperating clinicians that due to such advantages as noncontact and fast measurements as well as decreasing cost of instrumentation it would be interesting and profitable to check new clinical applications of this technology. Natural fields of such applications concern investigation of external parts of the human body, including scars, burns and other superficial wounds. We already applied IR-thermography in evaluation of numerous surgical procedures in cardiosurgery [2], burn diagnostics [3] and other applications [4] showing quality of applied medical procedures as well as the value of IR-technology in clinical practice. Still, one of untouched by quantitative technology fields is the problem of post-operative wound healing [5].

The aim of the research described in this presentation was to prove that thermal imaging may be an effective and quantitative tool in diagnostics of the state of a cardiosurgery postoperative wound. We applied thermal study to 
regular cardiosurgery patients of the Clinic of Cardiac \& Vascular Surgery GUMed according to the decision of the local ethical committee. Total number of patients who accepted participation to the research excided 400 . The study was divided into two phases - preliminary with the aim of development of measurement procedures and definition of advisable conditions of diagnostics - results of this phase were discussed in [1] - and the second stage, to prove clinical value of the proposed method.

The main result of this research is the proposal of a new quantitative method for classification of patients with a wound healing without complications and patients who should be treated with special care as the healing process is suspected.

\section{The method}

The proposed approach of thermal investigations may be explained based on Fig.1, where all basic elements of the experimental set are shown. Important is the RGB camera as till now visual inspection decided on qualitative clinical decisions. The IR-thermal camera is applied as the basic quantitative diagnostic instrument allowing registration of static temperature distribution at the surface of patients' skin as well as transient thermal processes after external thermal excitation to be applied. Here the system of two cryotherapy units driven automatically is shown. All registration is controlled by the main computer. The sequence of diagnostic procedures starts with registration of all optical and thermal data at the steady state, before operation. ADT - Active Dynamic Thermography experiment begins by starting blowing coolant till the moment that temperature at the selected Region of Interest - ROI - reaches the room temperature level. This moment is detected by the IR-camera. Then serial of IR images is registered during the period of time typically equal to 3 times of the cooling period. Body temperature at $\mathrm{ROI}$ is rising during this period due to natural internal heat flows. Important diagnostic information is extracted from thermal images as there is strong correlation of thermal data and wound healing processes. As the descriptor allowing quantitative evaluation of healing processes the second time constant of the two exponential thermal model is chosen. This parameter appears most sensitive and reliable for taking diagnostic decisions.

Such measurements are repeated at the day after operation and three days later, when normally a patient is discharged from a hospital for further rehabilitation at home. Comparison of thermal data taken after operation and at the discharge day allow for clear evidence if the healing process is regular or with complications. This allows for objective diagnostic decision and registration as important document in an electronic patient record.

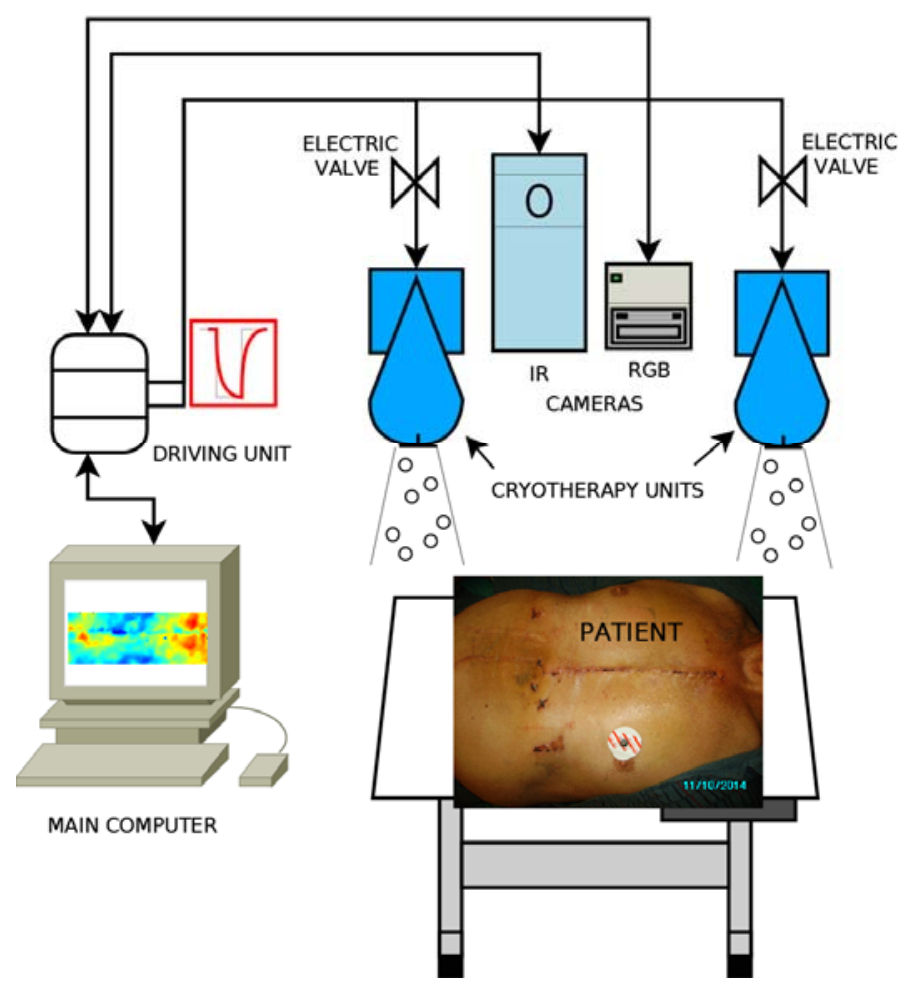

Fig. 1. Measurement set allowing thermal diagnostics of a post-operative wound 
Details of the instrumentation shown in Fig. 1 are discussed in the following paragraph. Specific role of cooling should be underlined. First, comparing with heating where optical, fully aseptic excitation is possible the main advantage of cooling is possibility of stronger signal generation. Optical heating should never exceed $42^{\circ} \mathrm{C}$ as higher temperature may be harmful. Taking into account that temperature at the surface of the skin typically is $35^{\circ} \mathrm{C}$, but at the hot spot even $39-40{ }^{\circ} \mathrm{C}$, signal after heating is strongly limited what not allows for receiving sufficient high $\mathrm{S} / \mathrm{N}$ signal to noise ratio. Cooling to the room temperature, typically $20^{\circ} \mathrm{C}$, gives reasonably better IR signal and improvement of S/N. The other even more important argument is determination of heat exchange conditions. This problem is discussed in detail in [6]. Cooling to room temperature assures lack of heat exchange at the environment in steady state conditions. Therefore at the beginning of the recovery phase only internal heat flows are possible, what allows for strong correlation of surface temperature transient processes with body internal physiology, decisive for healing processes.

\section{The instrumentation}

The measurement set shown in Fig.1 is very versatile allowing use of different IR and RGB cameras what is shown in Fig. 2, block diagram of the stand. In practice up to four cameras may be connected to the driving unit, and we checked possibility of stereo 3-D imaging in visual as well as in IR domains. Also we checked usability of different IR cameras, in Fig. 3 high quality camera with quantum well FPA detectors is shown. For the application we discuss in this paper in practice most of modern IR cameras allowing measurements of temperature of human body with resolution better than $0.1^{\circ} \mathrm{C}$ may be applied. Practical for clinical measurements is the set shown in Fig.4. with inexpensive room temperature bolometer FPA. We performed most of measurements using this arrangement. We also tried to use the stand shown in Fig. 5, using high performance air cooling system. The main advantage of this solution is handy situation comparing to the set from Fig. 4, where delivery of compressed $\mathrm{CO}_{2}$ bottles is necessary. Unfortunately for longer use special care of clean air filters to protect the wound is necessary. Also this stand is a bit noisy.

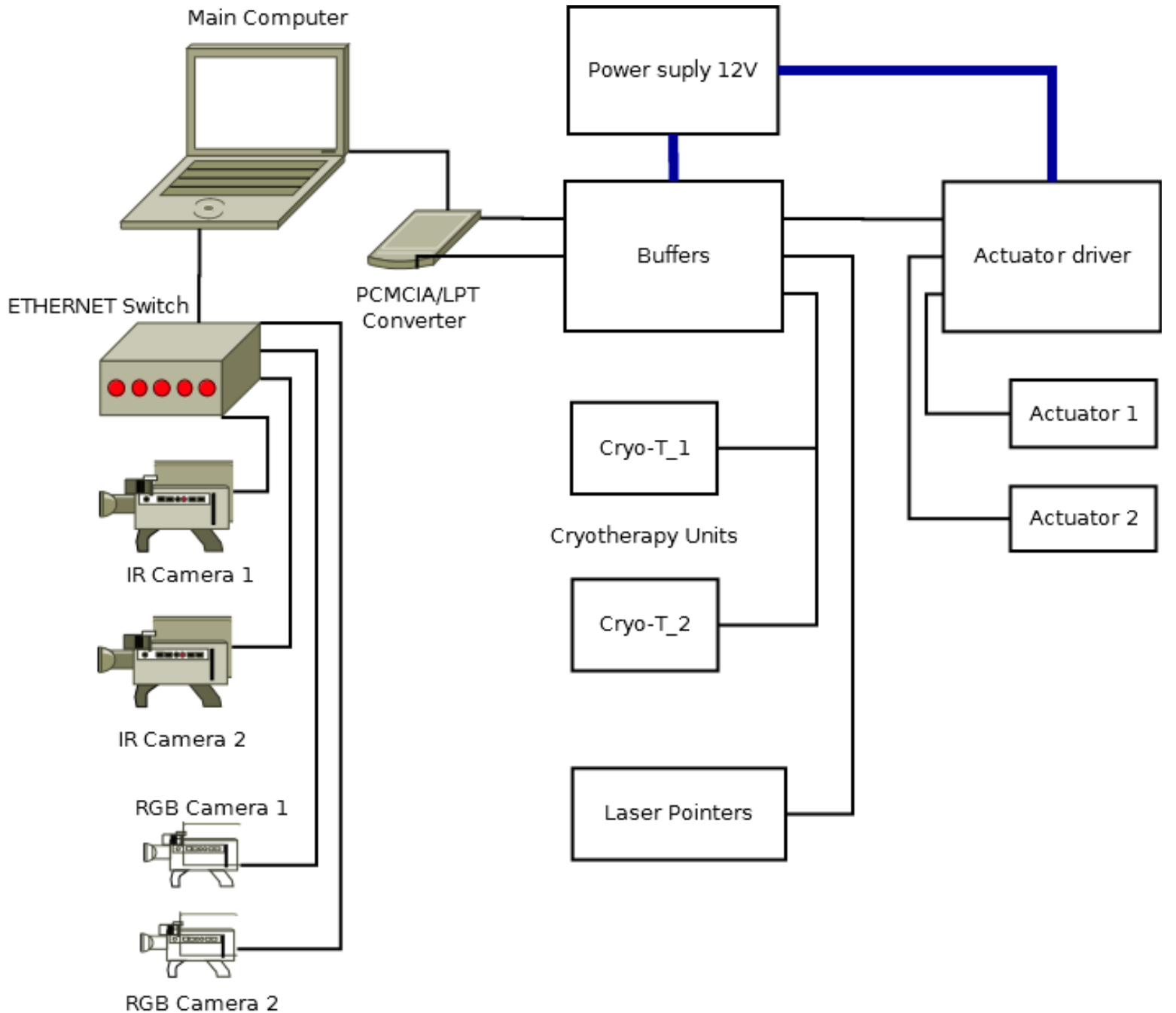

Fig. 2. Block diagram of the measurement set - configuration for 3-D experiments 


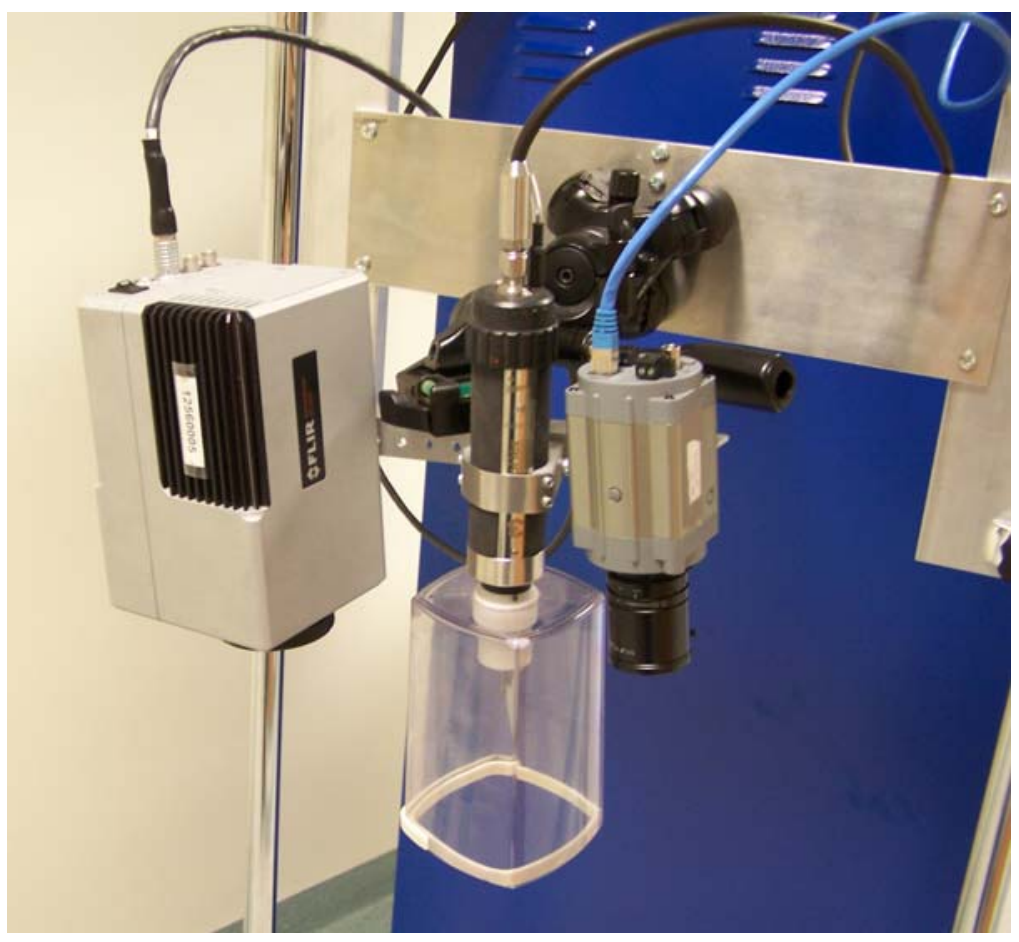

Fig. 3. Basic elements of the diagnostic set - from the left: the high performance cooled quantum well FPA IRcamera, the head of the cryotherapy cooling unit and the RGB camera

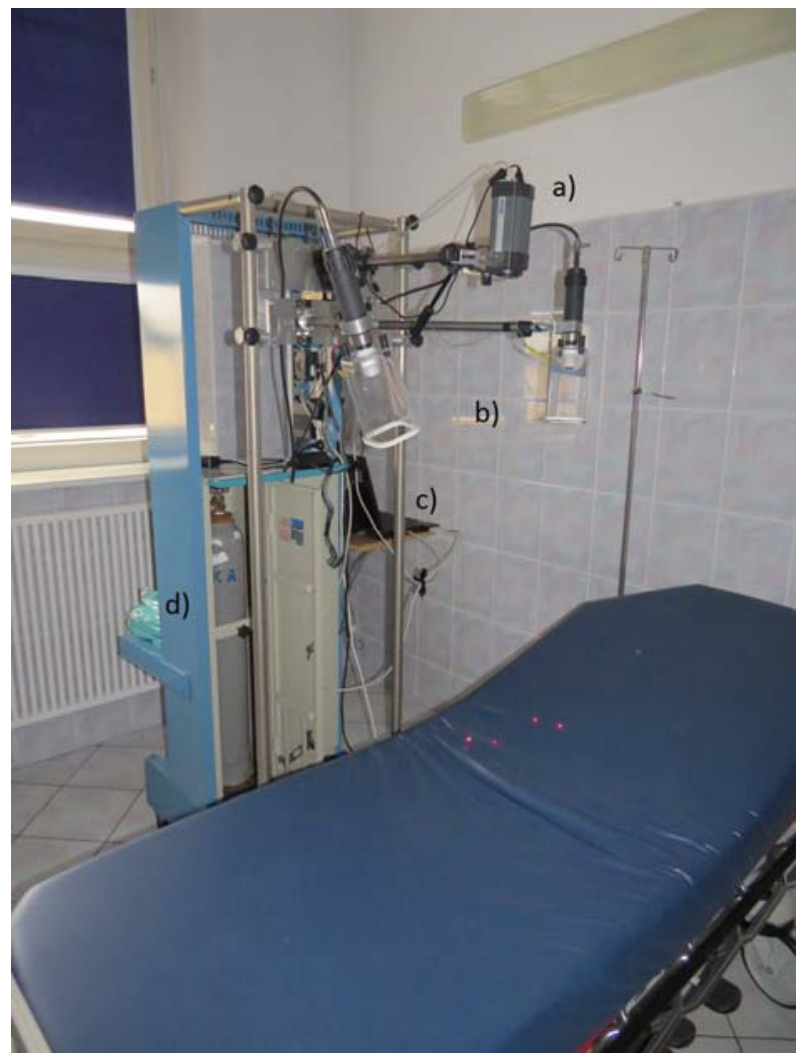

Fig. 4. The diagnostic set in a hospital environment: a) IR-camera with room temperature bolometer FPA; b) two cooling $\mathrm{CO}_{2}$ heads - red dots on the patient bed show the ROI to be cooled; c) a laptop as the driving-registration unit; d) a standard 19" frame to hold all elements of the system 


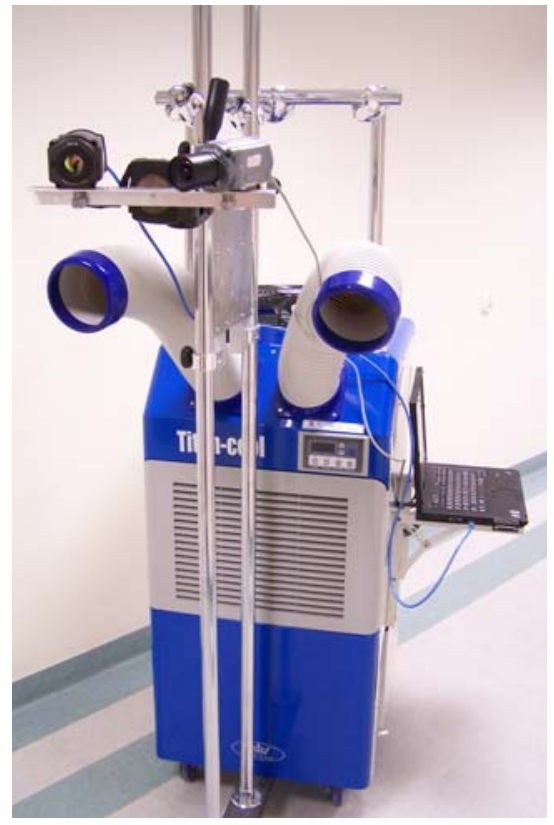

Fig. 5. The diagnostic set with a high power air cooler - IR-camera with room temperature bolometer FPA and RGB camera; two air cooling heads should be directed to the ROI to be cooled; the laptop as the driving-registration unit

\section{The software tools and results}

There are two levels of software tools - the basic one is for establishment of measurement conditions and execution of ADT measurements. This is fully automatic process after definition of basic measurement conditions. As an example in Fig. 6 the basic window of driving the system is shown. First, important conditions of IR-camera properties are set. Images - temperature distribution and visual one may be selected. After definition of a ROI one may observe the trace of temperature in a selected pixel or an averaged value in ROI. A single image or a set of serial images during chosen period of time may be registered. The results are collected in a patient database - electronic medical record. Measurements are repeated after the operation and whenever it is necessary. To make any diagnostic decision further data treatment and analysis is necessary.

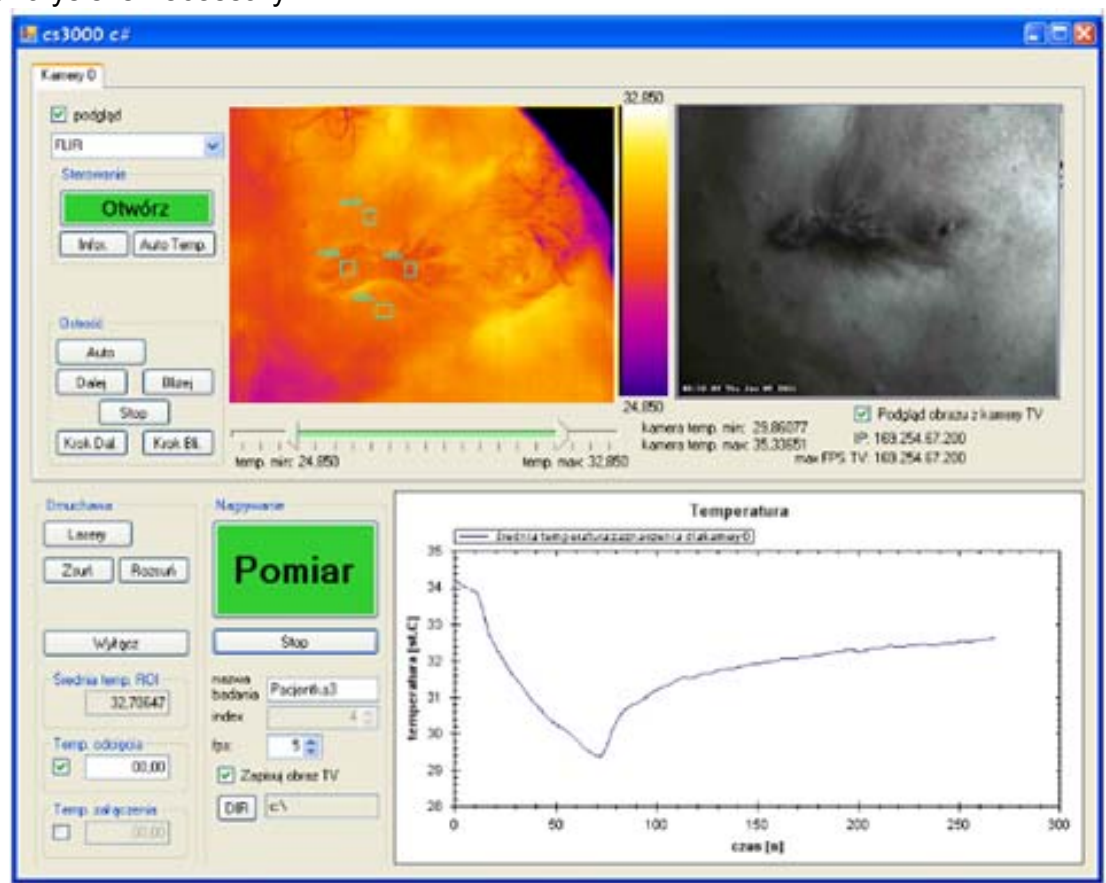

Fig. 6. The main page of the driving software - choice of IR-camera and RGB camera; determination of ROI; driving of laser pointers; plot of temperature in a given ROI 
Tools of the second level software are devoted to digital signal processing and image manipulation, including analysis of series of images according to ADT procedures. Mainly MATLAB procedures and for analysis of thermal processes the COMSOL software are applied. There are several packages of software prepared for data analysis. The first one is devoted to geometry corrections, matching of images taken in consecutive days of treatment and determination of the same ROI in all images. The next step may be denoising/filtration of images. Also matching of different modality images (mainly RGB, temperature, model parameter synthetic descriptors) may be useful in qualitative evaluation of the content. One of examples is shown in Fig. 7. Usually thermal images are of a low geometry resolution so matching with visual image allows for more precise interpretation of diagnostic content. As an example of matching images in Fig. 8 a color photograph and a thermogram with additional determination of the ROI and inserted parametric image of a descriptor, here distribution in ROI of the second time constant of the two exponent model are shown. Such approach may be very useful in qualitative evaluation of the content of images.

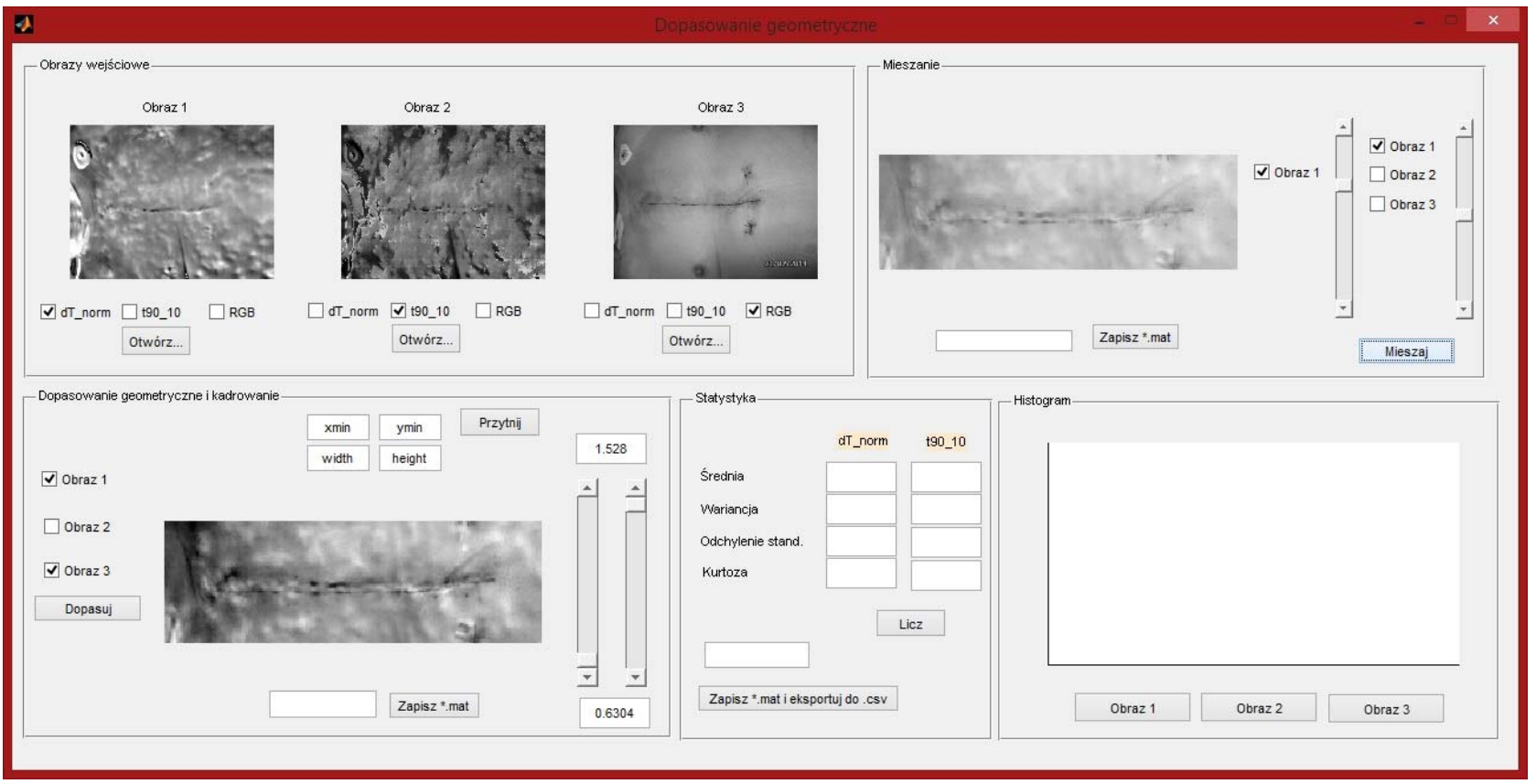

Fig. 7. One of pages showing result of ROI determination and transformation of images [7]

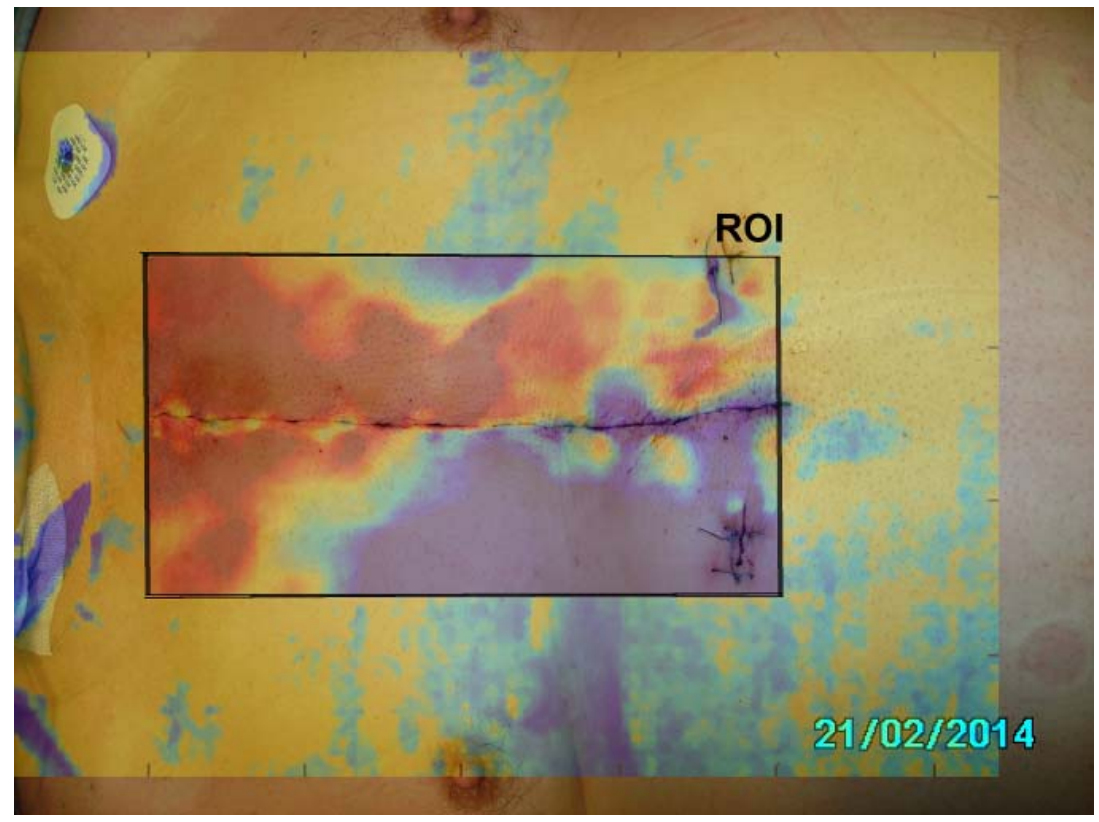

Fig. 8. An example of multimodality analysis with RGB, IR-thermal and parametric at the ROI images 
For quantitative analysis that is the main goal of the research we propose to discuss the example shown on Fig.9. It is the result of collecting and processing diagnostic images of a patient having complicated recovery after open heart cardiosurgery intervention. The data are collected at the day after operation (day 3) and three days later (day 6). The day 6 is taken intentionally as typically this is the day when a patient recovering without complication is discharged for further rehabilitation at home. Photographs and following thermal parametric images are shown. The applied classifier of diagnostic decision is the differential image of the second time constant of two exponent thermal model. For this classifier only the closest to the wound region of the ROI is presented at the decisive image. There are two rows of pixels averaged in segments +/- 5x10 along 200 pixel length covering the whole wound. Interpretation of the classifier: White complications; Gray - non decisive, hard to say; Black - healing properly, no complications. For comparison the diagnostic result of a patient with no complications is also presented.

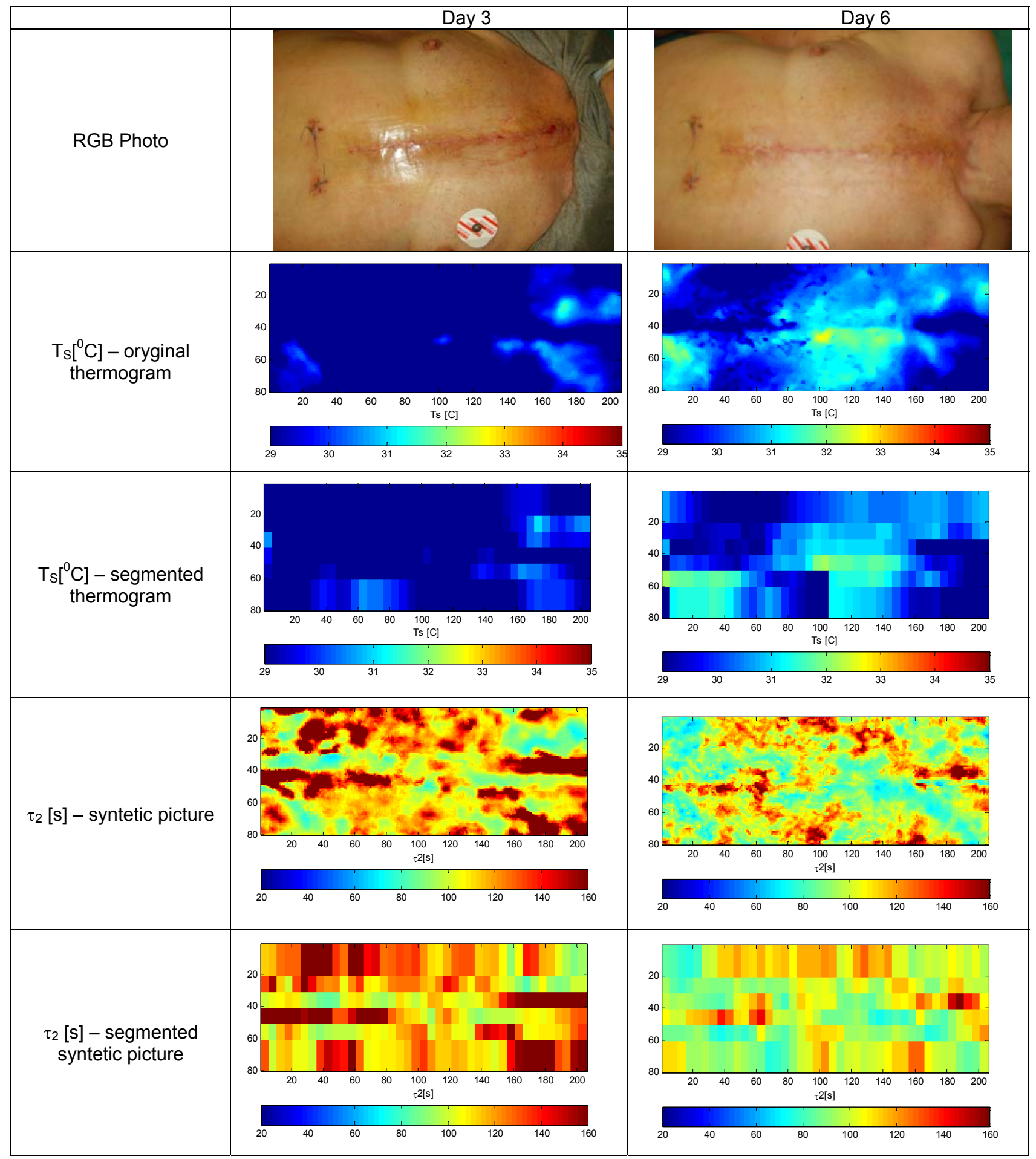




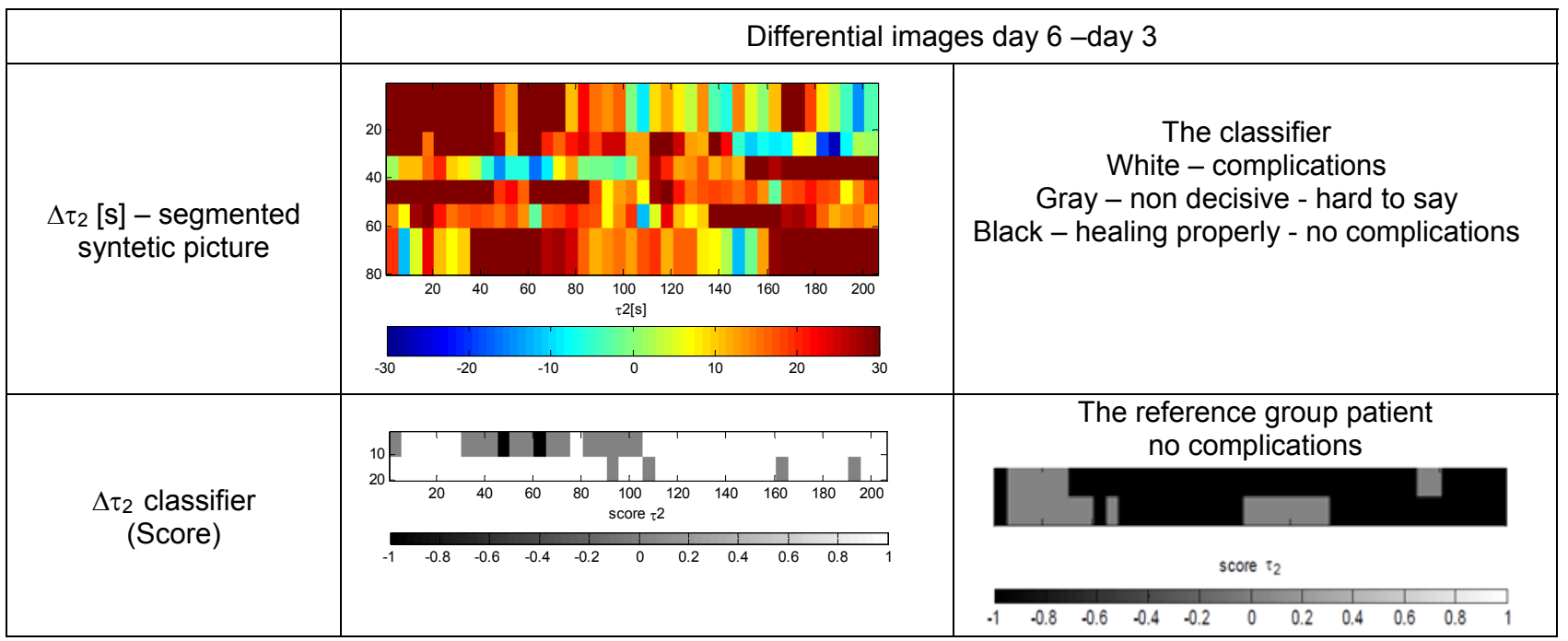

Fig. 9. Data treatment of patient

\section{Conclusions}

The results of the described project show that matching of different diagnostic modalities strongly improves possibility of proper understanding the content of medical diagnostic images. Also our long lasting cooperation in clinical environment of medical and technical staff proved that common research is very profitable for both sides.

The main outcome of this project is the proposal of a new in medical diagnostics method of quantitative evaluation of wound healing processes. This is a reliable tool allowing for objective quantitative description of the state of a post-cardiosurgery patient. One of important outcomes is preparation of a digital patient record which allows for objective evidence of the decision to keep a patient in the hospital or to allow him for further recovery and rehabilitation at home.

\section{Acknowledgements}

The work was co-financed by the Polish NCN research grant UMO-2011/03/B/ST7/03423 Development of new IR-imaging descriptors for objective evaluation of post-operative wound healing, and from the faculty WETI PG statute funds.

\section{REFERENCES}

[1] Nowakowski A., Siondalski P., Moderhak M., Kaczmarek M., Problems of cardiosurgery wound healing evaluation, Proc. QIRT, QIRT-2014-139pdf, p. 1-9, CD, Bordeaux, 2014.

[2] Nowakowski A., Kaczmarek M., Rogowski J., The Role of Thermal Monitoring in Cardiosurgery Interventions, in ed. M. Diakides, J.D. Bronzino, D.R Petereson, Medical Infrared Imaging - Principles and Practices, 17-1 - 1724, CRC Press, Taylor \& Francis Group, Boca Raton, 2013.

[3] Renkielska, A., Kaczmarek, M., Nowakowski, A., Grudziński, J., Czapiewski, P., Krajewski, A., Grobelny, I., Active dynamic infrared thermal imaging in burn depth evaluation, Journal of Burn Care and Research, 35(5):e294-303. doi: 10.1097/BCR.0000000000000059, 2014.

[4] Nowakowski A. Quantitative Active Dynamic Thermal IR-Imaging and Thermal Tomography in Medical Diagnostic. Ed. M. Diakides, J.D. Bronzino, D.R Peterson, Medical Infrared Imaging - Principles and Practices, 7-1 - 7-30, CRC Press, Taylor \& Francis Group, Boca Raton, 2013.

[5] Siondalski P. Algorytm leczenia powikłań gojenia się ran pooperacyjnych i ropnego zapalenia śródpiersia po operacjach kardiochirurgicznych [Algorithm dealing with the treatment of healing wound complications and mediastinitis after cardiac surgeries]. Annales Academiae Medicae Gedanensis. 39:303-4135, supl. 2, 2009.

[6] Renkielska A., Nowakowski A., Kaczmarek M., Ruminski J., „Burn depths evaluation based on active dynamic IR thermal imaging-A preliminary study”, Burns, Vol. 32 (7), 2006, s. 867-875.

[7] Mongard N., Sadowska S., GUI for translation and rotation compensation in thermal images, engineering diploma project, TUG, Gdansk, 2014. 